\title{
Electrostatic Frequency Tuning of Bulk Acoustic Wave Disk Gyroscopes
}

\author{
Madan Parajuli, Guillermo Sobreviela, Ashwin A. Seshia \\ Nanoscience Centre, Department of Engineering, University of Cambridge, Cambridge, UK \\ Email:mp912@cam.ac.uk
}

\begin{abstract}
Bulk acoustic wave gyroscopes have been researched for potential benefits such as immunity to shock and vibration and the high $Q$ factors achievable with the bulk modes. This paper outlines an approach to address mode matching in bulk acoustic wave (BAW) disk gyroscopes using electrostatic frequency tuning. Electrostatic frequency tuning is achieved by varying the potential difference between the body of the resonator and electrodes surrounding the disk resonator. Tuning of the frequencies of both drive and sense modes is demonstrated in this work as a means to achieving mode matching. The experimental results are also compared to COMSOL simulations reporting mode matching.
\end{abstract}

Keywords-MEMS, Gyroscopes, bulk acoustic wave sensors, mode matching

\section{INTRODUCTION}

The simplest abstraction of a single-axis Coriolis vibratory rate gyroscope is the lumped element two degrees-of-freedom (2-DOF) mass-spring-dashpot model shown in Fig. 1. The schematic of this single-axis gyroscope models a drive mode (ideally aligned with the drive direction (x)) and a sense mode (ideally aligned with the sense direction (y)) as shown schematically. Further, the lumped spring elements are subdivided into two components, representing the mechanical and electrical spring terms respectively. As a result, the natural frequency of the drive mode $\left(f_{x 0}\right)$ and sense mode $\left(f_{y 0}\right)$ can be written as:

$$
\begin{aligned}
& f_{x 0}=\frac{1}{2 \pi} \sqrt{\frac{k_{x m}-k_{x e}}{m}} \\
& f_{y 0}=\frac{1}{2 \pi} \sqrt{\frac{k_{y m}-k_{y e}}{m}}
\end{aligned}
$$

where $m$ is the proof mass, $k_{x m}$ and $k_{y m}$ are mechanical stiffnesses in the drive direction and the sense direction respectively, and $k_{x e}$ and $k_{y e}$ are electrical stiffnesses in the drive and sense directions. A significant design challenge for MEMS gyroscopes is to achieve low-noise and high stability measurements. A possible approach to addressing low noise and high output stability in MEMS gyroscopes is to employ mode-matched operation [1], [2] where the frequencies of the drive and sense modes are precisely matched. Maintaining the condition of mode matching during gyroscope operation is preferable as this maximizes the mechanical scale factor of the device enabling low-noise operation. High-stability measurements are achieved under low dissipation conditions when the mode-matched condition is precisely maintained over the time course of the measurements. The dynamics under mode-matched conditions can be abstracted as two high-Q oscillators coupled via the Coriolis effect where energy is pumped back and forth between drive and sense modes.

Higher-order elliptical modes of bulk acoustic wave (BAW) disk gyroscopes fabricated in (100) silicon substrates exhibit degeneracy [3], [4] as well as high quality factors providing an excellent candidate for mode-matched operation. However, as previously outlined fabrication tolerances [5], material anisotropy [4], and practical considerations associated with anchoring arrangements can result in a non-zero frequency split between these modes in actual devices. In such cases, electrostatic frequency tuning can be employed to achieve mode matching [5]. However, it can be difficult to compensate for even a small mismatch in the natural frequencies due to the high mechanical stiffnesses associated with the BAW modes. A systematic approach to mode matching using electrostatic frequency tuning also relies on a thorough understanding of alignment of the mode shape with respect to the peripheral electrodes so that the tuning can be carried out by devising an appropriate strategy to define the magnitude of the tuning voltage(s) and choice of electrode(s).

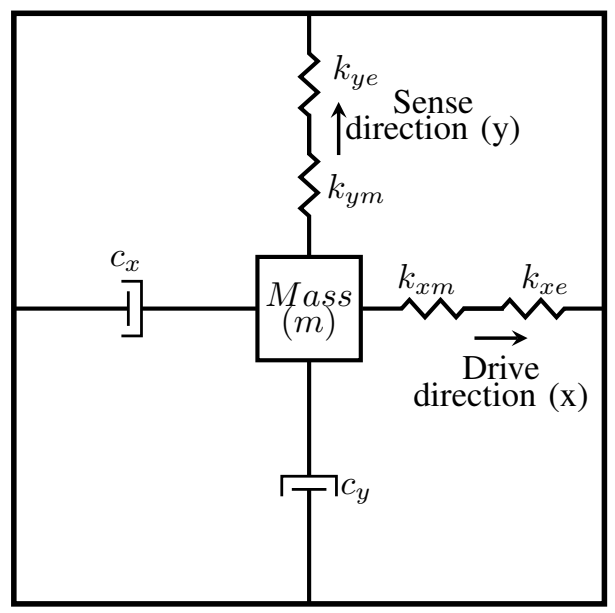

Fig. 1: A 2-DOF mass-spring-damper model for a single-axis Coriolis rate gyroscope.

\section{DEVICE}

The device-under-test in this study is a vacuum packaged micromachined disk gyroscope fabricated in a silicon-on- 
insulator (SOI)MEMS process employing wafer-level vacuum encapsulation [5], [6]. Fig. 2 shows the optical micrograph of the device. The disk has a radius of $3 \mathrm{~mm}$ and a thickness of $40 \mu \mathrm{m}$. The disk is surrounded by 12 identical electrodes and it employs edge anchoring. The modes of interest for the studies in this paper are the secondary degenerate modes. The evolution of these mode shapes with added stiffness perturbation is modelled using finite element analysis (COMSOL) and the results are illustrated in Fig. 3. These simulations are employed as a means of understanding the evolution of the response with perturbations due to geometric imperfections, material anisotropy and anchoring arrangements, as well as to model the effect of stiffness variations due to electrostatic loads.

The devices are experimentally characterised in an openloop configuration first. Fig. 4 plots the exponential decay of the output voltage for a representative device during a ringdown experiment. The natural frequency for the secondary elliptical mode of this device is $976.8 \mathrm{kHz}$ and the ringdown time for the first mode (lower frequency mode) $(\tau)$ is $0.57 \mathrm{~s}$, corresponding to a quality factor of $1.74 * 10^{6}$. A key figure of merit is related to the f.Q product. For the specific device-under-test, this value is $1.7 * 10^{12}$ which benchmarks favourably with respect to the state-of-the-art.

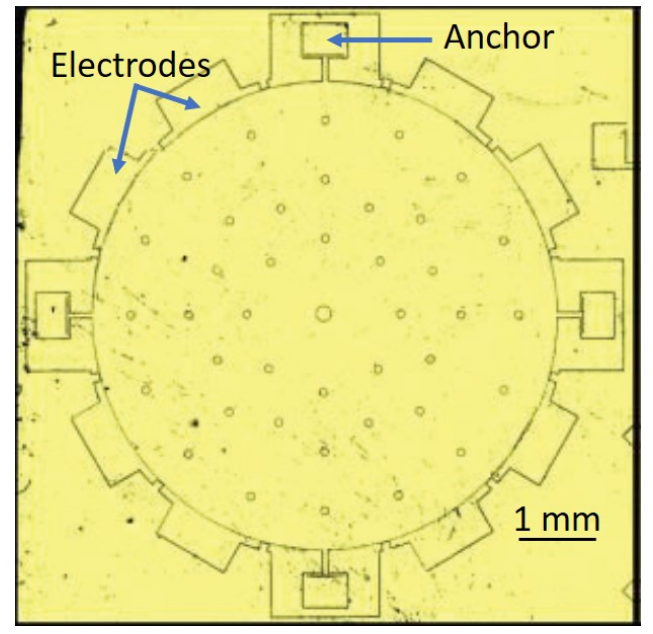

Fig. 2: Optical micrograph of the MEMS device [6].

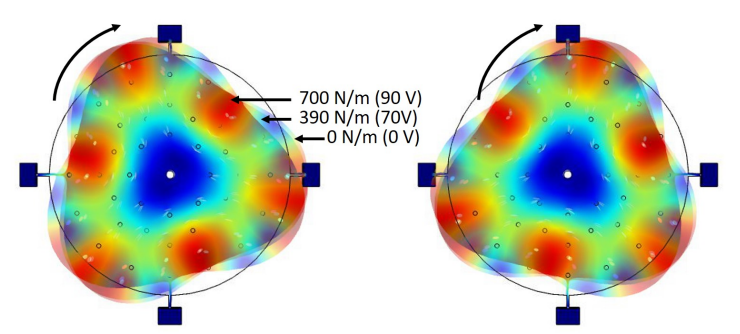

Fig. 3: The evolution of secondary degenerate modes with added stiffness perturbation.

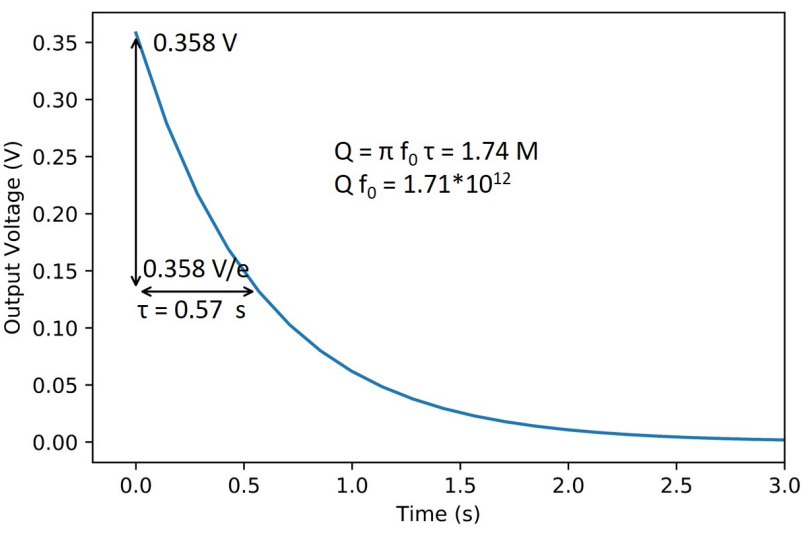

Fig. 4: Measured ring down response.

\section{RESULTS}

The experimental setup shown in fig. 5 is used to drive, sense and tune the frequency of the near degenerate modes of two devices fabricated in the same run. The devices were all biased with a polarisation voltage of $90 \mathrm{~V}$ DC and driven by a $0.5 \mathrm{Vrms}$ actuation signal. A HF2LI lock-in amplifier was used to record the response for open-loop measurements. Fig. 6 provides a schematic view of the device and surrounding electrodes. The tuning electrodes must be aligned to the antinodes of the mode to vary the electrical stiffness. The mode shapes were determined by analysing the amplitude and phase responses from all the electrodes while employing the $\mathrm{C} 5, \mathrm{C} 7$ and $\mathrm{C} 9$ electrodes for driving the device. The relevant mode shapes of Device I are represented by triangles, as shown in fig. 6 . The triangles represent both the in-phase and out-ofphase displacements and the vertices of the triangles represent the antinodes of both the first mode and the second mode. The first mode of Device II is similar to the second mode of Device I and vice versa.

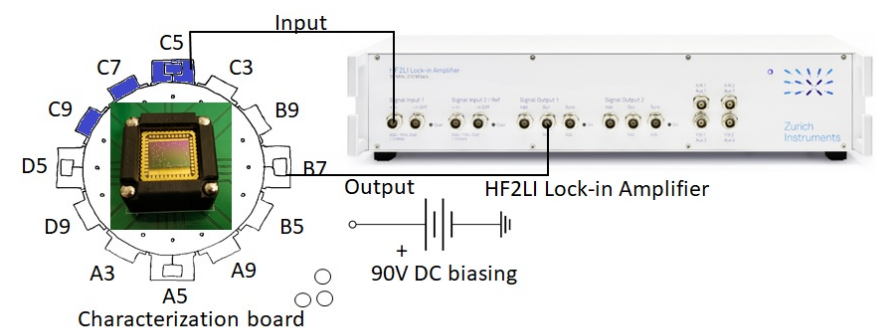

Fig. 5: Experimental setup.

Three approaches can be used to achieve mode matching:

a. Tuning the resonant frequency of the first mode;

b. Tuning the resonant frequency of the second mode;

c. Tuning both modes simultaneously.

In the experiments described in this paper, the first two approaches listed above have been employed. 

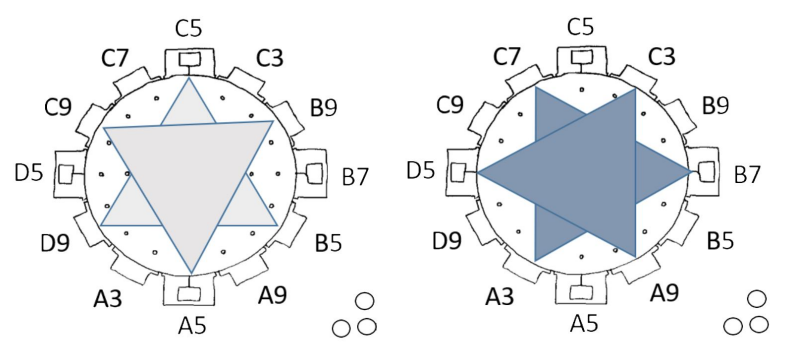

Fig. 6: First (left) and second (right) modes of Device I.
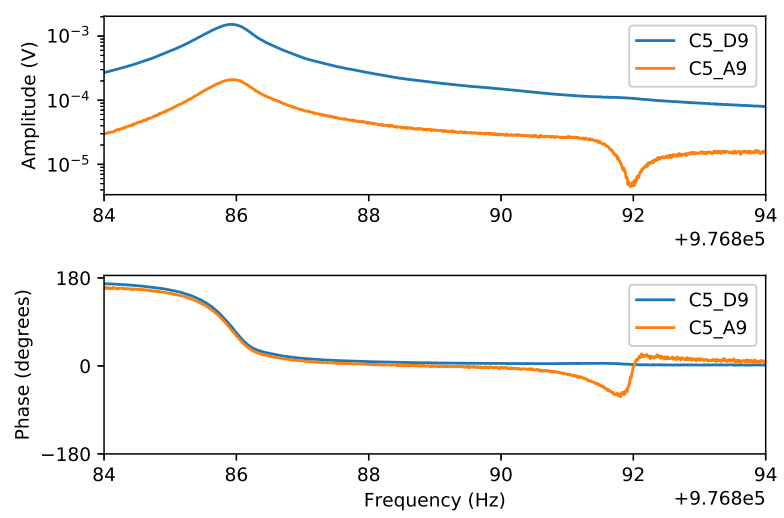

(a)
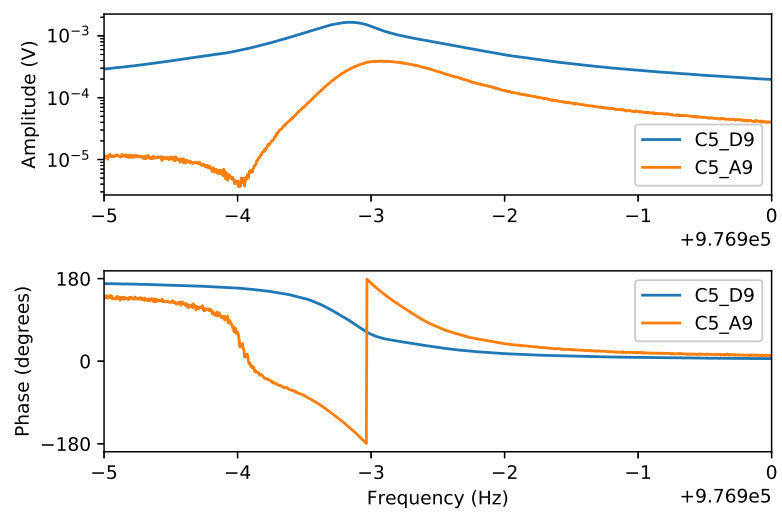

(b)
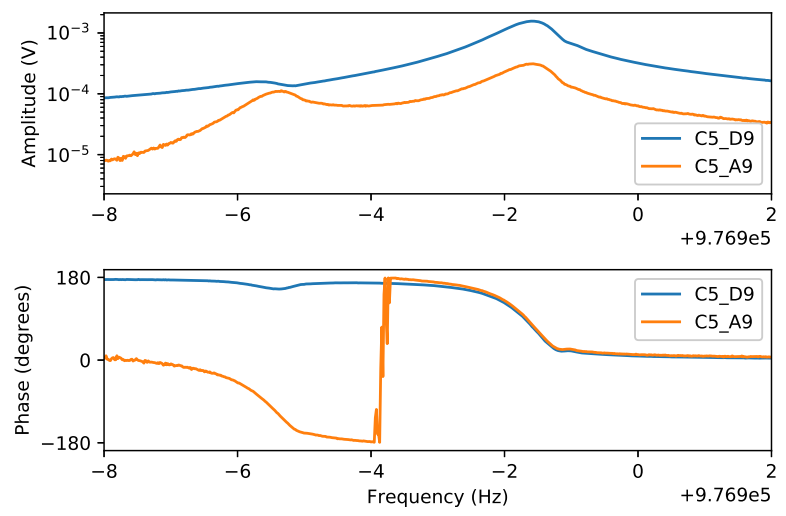

(c)

Fig. 7: Amplitude and phase response at D9 and A9 (a) before mode matching, (b) during mode matching (c) after mode matching (mode crossing).
In Device I, the resonant frequency of the first mode was increased to match the resonant frequency of the second mode. By supplying a positive tuning voltage on the (initially grounded) electrodes aligned to the antinodes of the first mode, the corresponding electrical stiffness is reduced, increasing the resonant frequency towards matching the resonant frequency of the second mode.

In Device II, the resonant frequency of the second mode was decreased to match the resonant frequency of the first mode. A negative tuning voltage on electrodes aligned to the antinodes of the second mode increases the electrical stiffness, decreasing its resonant frequency to match with the resonant frequency of the first mode.

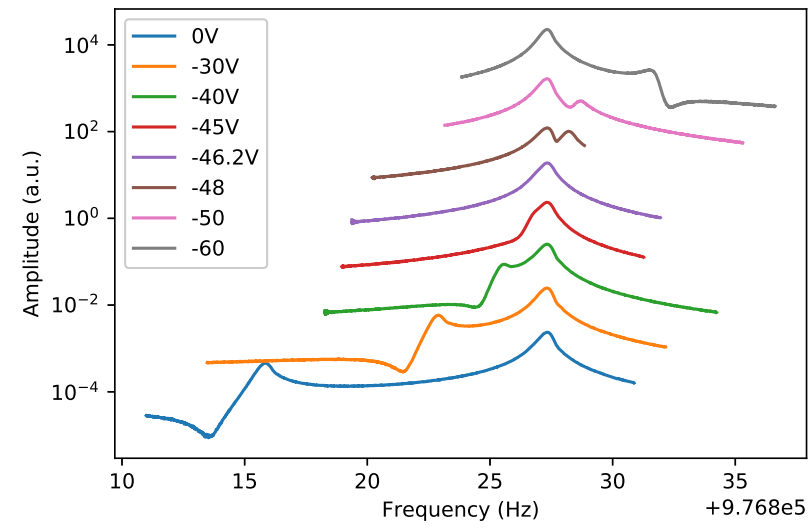

(a)

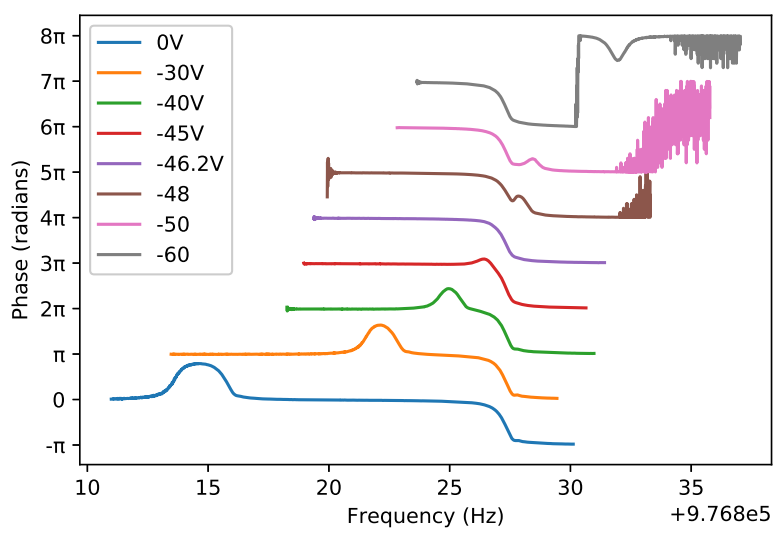

(b)

Fig. 8: (a) Amplitude and (b) phase response for different tuning voltages applied to A3, the curves are displaced (a) one order of magnitude up and (b) $2 \pi$ up progressively as the tuning voltage decreases.

The mode matching results achieved on Device I and Device II are summarized in fig. 7 and fig. 8 respectively. In Device $\mathrm{I}$, when $0 \mathrm{~V}$ is applied to the selected tuning electrodes, the frequency split between the two modes is $6 \mathrm{~Hz}(6.14 \mathrm{ppm})$ (fig. 7a). However, when the $24.6 \mathrm{~V}$ tuning voltage is applied, 
the two peaks are aligned, achieving mode matching (fig. 7b). Further increment of tuning voltage results in mode crossing (fig. 7c).

In Device II, the initial frequency split of $12 \mathrm{~Hz}(12.28$ ppm) at $0 \mathrm{~V}$ has gradually decreased to less than $1 \mathrm{~Hz}$ (1.02 ppm) at -46.2 V. Fig. 8 shows the amplitude and phase response at different tuning voltages. Similar to Device I, further decrement in the negative tuning voltage resulted in mode crossing.

The plots of the frequency difference between two modes with respect to tuning voltage for Device I and Device II are presented in fig. 9a and fig. 9b. The results are broadly consistent with COMSOL simulations as shown in fig. 10.

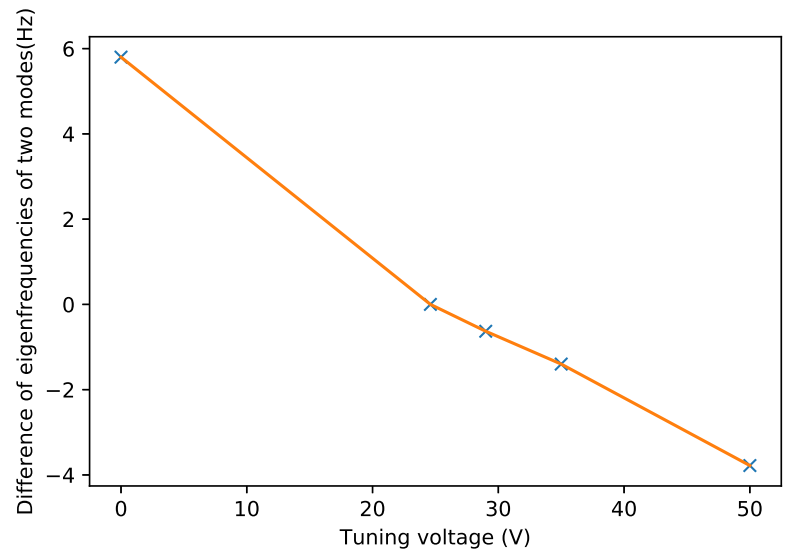

(a)

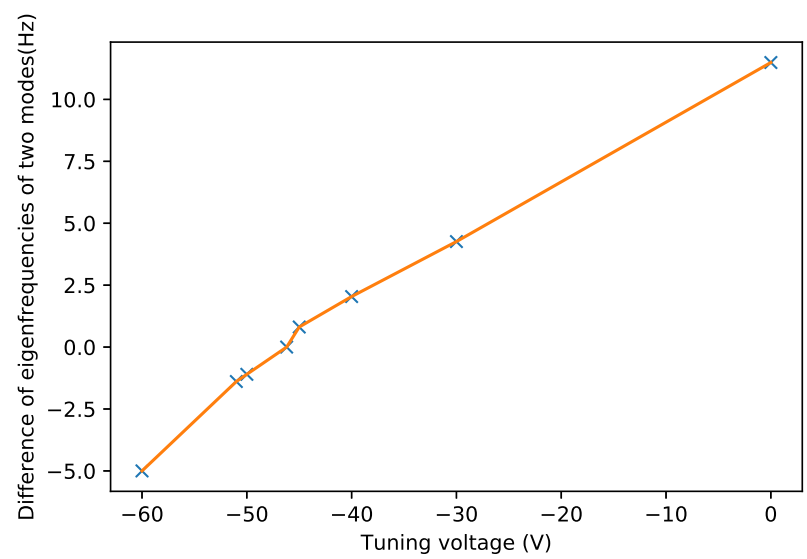

(b)

Fig. 9: A plot of the frequency split versus tuning voltage for (a) Device I (b) Device II

\section{SUMMARY}

This paper presents experimental results demonstrating electrostatic frequency tuning towards mode matching in bulk acoustic wave disk gyroscopes. Finite element simulations are employed to gain insight into the evolution of mode shapes and natural frequencies of the secondary elliptical modes in

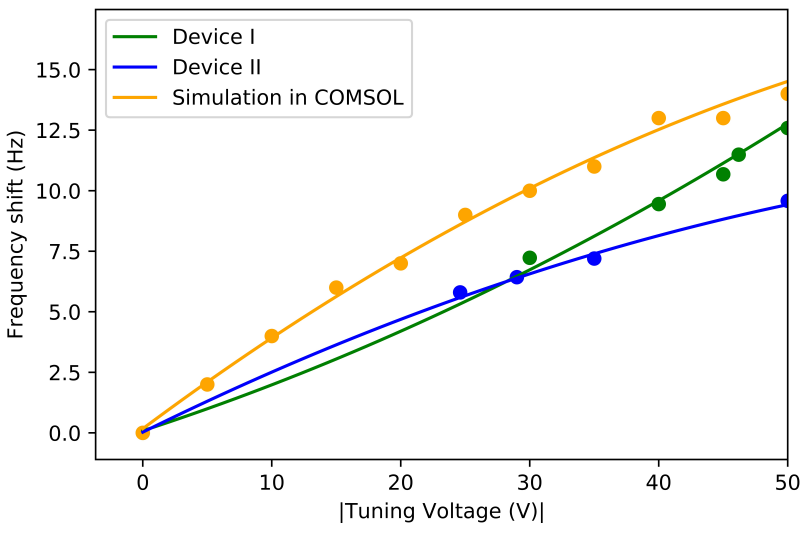

Fig. 10: Comparison of the frequency split between the drive and sense mode natural frequencies for varying tuning voltages for Device I and Device II compared to COMSOL simulations.

the disk when subject to induced stiffness perturbations. Two specific vacuum packaged disk gyroscopes are experimentally characterized. These devices demonstrate initial frequency splits of $6 \mathrm{~Hz}$ and $12 \mathrm{~Hz}$ which are subsequently reduced to less than $1 \mathrm{~Hz}$ with the application of specific tuning voltages applied to selected electrodes. The natural frequencies of both drive and sense modes can be separately tuned to achieve mode matching and the most appropriate strategy subsequently be employed based on initial characterisation of the associated modal responses. Future work is currently addressing the electronics build to enable full gyroscope characterisation under mode-matched conditions.

\section{ACKNOWLEDGMENT}

This work was supported by funding from Silicon Microgravity and Innovate UK.

\section{REFERENCES}

[1] I. P. Prikhodko, J. A. Gregory, W. A. Clark, J. A. Geen, M. W. Judy, C. H. Ahn, and T. W. Kenny. Mode-matched mems coriolis vibratory gyroscopes: Myth or reality? In 2016 IEEE/ION Position, Location and Navigation Symposium (PLANS), pages 1-4, 2016.

[2] C. Acar, A. R. Schofield, A. A. Trusov, L. E. Costlow, and A. M. Shkel. Environmentally robust mems vibratory gyroscopes for automotive applications. IEEE Sensors Journal, 9(12):1895-1906, 2009.

[3] D. E. Serrano, M. F. Zaman, A. Rahafrooz, P. Hrudey, R. Lipka, D. Younkin, S. Nagpal, I. Jafri, and F. Ayazi. Substrate-decoupled, bulkacoustic wave gyroscopes: Design and evaluation of next-generation environmentally robust devices. Microsystems \& Nanoengineering, 2(1):1-10, 2016.

[4] X. Wei and A. A. Seshia. Analytical formulation of modal frequency split in the elliptical mode of scs micromechanical disk resonators. Journal of Micromechanics and Microengineering, 24(2):025011, 2014.

[5] X. Zou, C. Zhao, and A. A. Seshia. Edge-anchored mode-matched micromachined gyroscopic disk resonator. In 2017 19th International Conference on Solid-State Sensors, Actuators and Microsystems (TRANSDUCERS), pages 2183-2186, 2017.

[6] G. Sobreviela, X. Zou, C. Zhao, M. Pandit, and A. A. Seshia. An ultrahigh-quality factor silicon disk resonator. In 2019 20th International Conference on Solid-State Sensors, Actuators and Microsystems Eurosensors XXXIII (TRANSDUCERS EUROSENSORS XXXIII), pages 527-530, 2019. 